\title{
Visual working memory organization is subject to top-down control
}

\author{
Amanda E. van Lamsweerde ${ }^{1,2} \cdot$ Melissa R. Beck $^{1} \cdot$ Jeffrey S. Johnson $^{2}$
}

Published online: 23 November 2015

(C) Psychonomic Society, Inc. 2015

\begin{abstract}
The limited capacity of visual working memory (VWM) can be maximized by combining multiple features into a single representation through grouping principles such as connection, proximity, and similarity. In this study, we sought to understand how VWM organizes information by investigating how connection and similarity cues are used either alone or in the presence of another grouping cue. Furthermore, we examined whether the use of one cue over another is within volitional control. Participants remembered displays of objects that contained no grouping cues, connection cues only, similarity cues only, or both connection and similarity cues. We found that it is possible to use either connection or similarity cues, although connection cues tend to dominate if the cues are in conflict with one another. However, it is possible to flexibly use either similarity or connection cues if both are present, depending on the task goals.
\end{abstract}

Keywords Visual working memory · Short-term memory · Perceptual organization

Visual working memory (VWM) can represent a small amount of information (three to four units; Luck \& Vogel, 1997) over a short period of time (several seconds; Zhang \& Luck, 2009). However, several features (e.g., red, circle) can

Electronic supplementary material The online version of this article (doi:10.3758/s13423-015-0976-3) contains supplementary material, which is available to authorized users.

Amanda E. van Lamsweerde amanda.vanlamsweerde@ndsu.edu

1 Louisiana State University, Baton Rouge, LA, USA

2 North Dakota State University, Dept. 2765, P.O Box 6050, Fargo, ND 58108, USA be grouped together as a single unit in VWM in order to maximize capacity (Luck \& Vogel, 1997). This feature grouping can be achieved via multiple perceptual-grouping principles, such as connection (Luck \& Vogel, 1997; Xu, 2006), proximity (Woodman, Vecera, \& Luck, 2003), or feature similarity (Peterson \& Berryhill, 2013). However, it is unclear how VWM is organized when two cues conflict, or to what extent this organization is under top-down control. We propose that some cues are likely to dominate over others, depending on the relative strengths of the perceptual cues, but that it should be possible to flexibly switch between grouping strategies, depending on the task goals.

Connection is a powerful cue that allows two features to be perceptually grouped, especially if the connection results in uniform visual properties such as a continuous color or texture (Palmer \& Rock, 1994; Watson \& Kramer, 1999). In VWM, the connection cue is easily used to increase the number of remembered features (Luck \& Vogel, 1997; Xu, 2002). Connections of some features (e.g., color and shape) can improve performance when the connected features form distinct parts (Xu, 2002, 2006; but see Davis \& Holmes, 2005), although the connection benefit is greater if the connected features share a continuous surface feature (Delvenne \& Bruyer, 2004; $\mathrm{Xu}, 2002)$. An exception to this is color-color connections, which consume as much capacity as two spatially separated colors (Delvenne \& Bruyer, 2004; Olson \& Jiang, 2002; Wheeler \& Treisman, 2002; Xu, 2002). This possibly occurs because the color boundaries impair the perception of a whole, which necessitates creating a new item representation (or object file) in VWM (Goldfarb \& Treisman, 2011).

Feature similarity allows spatially separate items to be perceptually grouped on the basis of shared features (Wertheimer, 1938). Attention spreads automatically across similar items, as it does across objects (Erlikhman, Keane, Mettler, Horowitz, \& Kellman, 2013; Kasai, Moriya, \& Hirano, 2011; Wannig, 
Stanisor, \& Roelfsema, 2011). Feature similarity can also be used to increase VWM capacity, at least for color: A greater number of spatially disconnected items are remembered if some of those items are of the same color (Peterson \& Berryhill, 2013).

However, not all grouping cues are equal in strength (Schmidt \& Schmidt, 2013); rather, some cues tend to dominate over others. For example, perceptually grouping by uniform connectedness is faster than grouping by shape similarity (Han, Humphreys, \& Chen, 1996), and proximity dominates over feature similarity when they are in conflict (Han, 2004). Likewise, in Experiment 1 of this study, we examined the fates of multiple grouping cues in VWM when these cues were in conflict. Given the strength of proximity over similarity in perception, we predicted that connection would likely be a stronger grouping cue than feature similarity.

Furthermore, even though some grouping strategies may take precedence over others, it is possible to use top-down control to bias the perception of a group. For example, the reaction time to a target pair is faster if both targets are part of the same perceptual group (via color similarity, proximity common region, uniform connectedness, or element connectedness); however, this grouping effect is reduced or eliminated if participants know that it is unlikely that the target pair will appear within a group (Beck \& Palmer, 2002). This suggests that it is possible to use top-down knowledge to influence the perception of a group. Likewise, the purpose of Experiment 2 was to determine whether the use of a grouping strategy in VWM is under top-down control.

\section{Experiment 1}

In Experiment 1, participants detected changes to displays that contained no grouping cues, feature similarity cues only, connection cues only, or both cue types (in conflict). Displays contained one of three stimulus types (color-only, shape-only, or color-and-shape stimuli), because different groupings may occur for different stimulus types. Connections of color and shape, but not of two colors, can be grouped in VWM (Wheeler \& Treisman, 2002), and similarity grouping has only been established with color (Peterson \& Berryhill, 2013). In Experiment 1, we established that groupings can also be created on the basis of shape similarity, and determined how VWM is organized for each stimulus type when similarity and connection cues conflict.

\section{Method}

\section{Participants}

In all, 70 undergraduate students at Louisiana State University participated for credit in their undergraduate psychology courses; one of the participants in the color-and-shape condition was omitted due to an error that caused the program to end prematurely, for a total of 69 participants ( 47 female, 22 male, average age $=20.06$ years). All students reported normal color vision, and 64 reported normal or corrected-to-normal vision. Twenty-six of the participants were assigned to the color-only condition, 22 to the shape-only condition, and 21 to the color-and-shape condition.

\section{Stimuli}

Features Eight colors (cyan, orange, red, pink, blue, purple, brown, and green) and eight shapes were used (Fig. 1). Each feature subtended $1.4 \mathrm{deg}$ of visual angle from the monitor to the edge of the table. All shapes were symmetrical about the $y$ axis and were of equal width at the base, so any two shapes (one upright, one inverted) could be combined.

Stimulus types Three stimulus types were used (Fig. 1). Colored squares were used for the color-only stimuli, so that participants detected only color changes. For the shape-only stimuli, participants detected only shape changes to black shapes. Half of the shapes in a display were upright and half were inverted, with the constraint that the same shape was never both upright and inverted. The color-and-shape stimuli consisted of two vertically stacked identical shapes, one upright and one inverted. The resulting shape was filled with a solid color when connected.

Cue display types Four cue displays were used (no-cue, connection-only, similarity-only, two-cue), all of which contained six task-relevant features. When similarity cues were absent (no-cue and connection-only displays), all features in the displays were unique. When connection cues were absent (nocue and similarity-only displays), all six features were spatially separated. In the two-cue displays, two identical items were never presented; for example, a display could contain a red oval, a red diamond, and a green oval, but not two green diamonds.

\section{Procedure}

Participants viewed a memory display for $500 \mathrm{~ms}$ (Fig. 2), followed by a $900-\mathrm{ms}$ delay, and then a test display until a response was given. On half of the trials, one of the six features changed from study to test. Participants indicated whether or not a change had occurred by pressing " $\mathrm{z}$ " or "/" on a keyboard. They completed 256 trials (half change and half no-change), except in the color-and-shape condition, in which they completed 512 trials, so that color and shape changes could be analyzed separately (there were no differences across change types, so all results are presented with color and shape performance collapsed). 


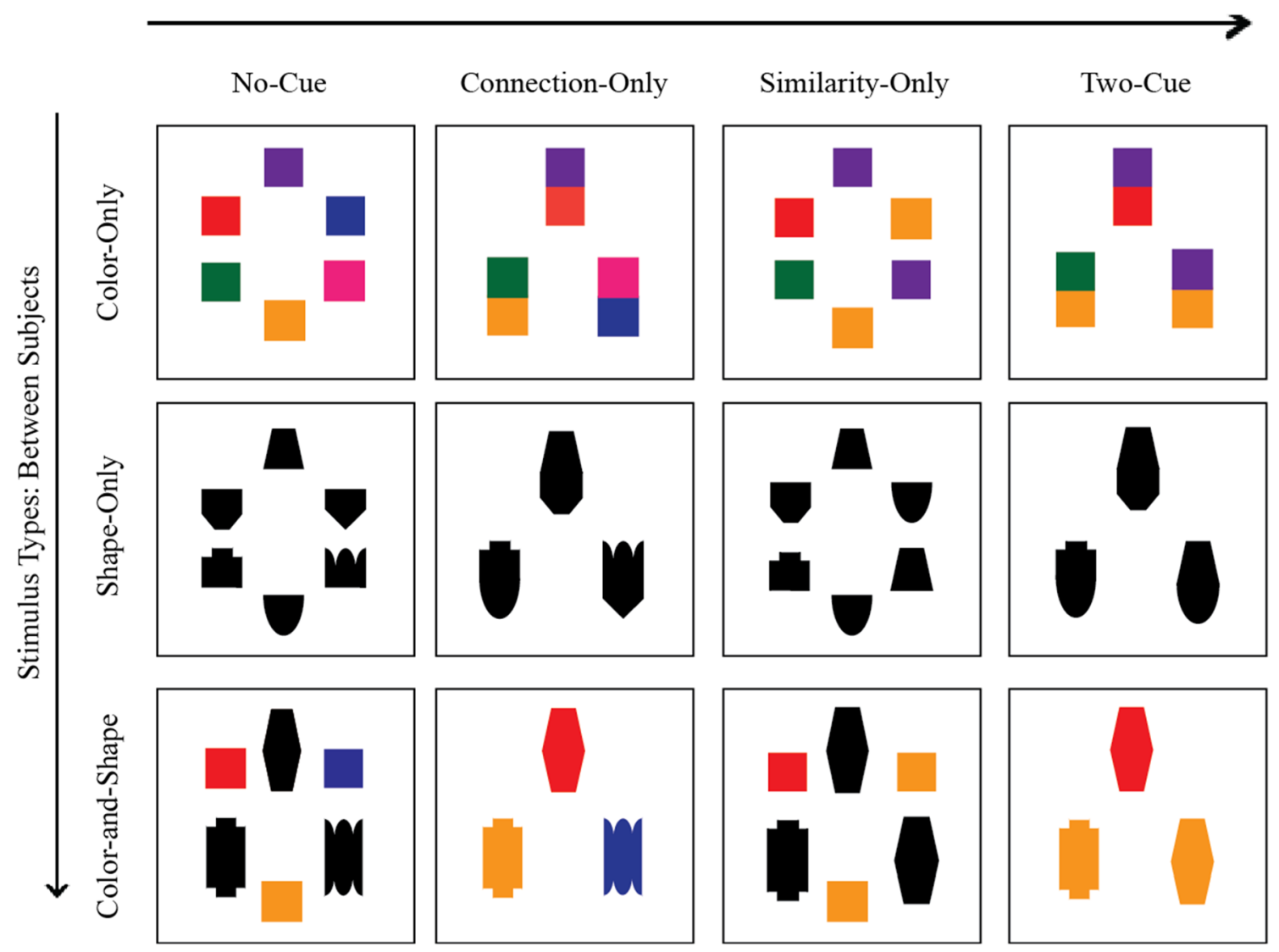

Fig. 1 All shapes (a) and all possible stimulus types and display types (b). Each feature combination is represented in the rows, and all cue display types are represented in the columns. All six features were presented in separate locations when there were no connection cues (no-cue and similarity-only displays). When the features were connected (connection-only and two-cue displays), two features were stacked vertically (with the constraint that two identical features were never connected), except for the color-and-shape stimuli. Objects were

Participants completed four blocks of trials, one for each cue display type, in counterbalanced order.

\section{Results}

For each feature combination, proportions correct were analyzed with a repeated measures analysis of variance (ANOVA) on the Cue Display Type factor (no-cue, connection-only, similarity-only, and two-cue), which was significant for all stimuli (Table 1). To specifically determine how cues affected performance, the ANOVAs were followed by four comparisons, presented as one-sample $t$ tests of difference scores. Difference scores were created for each cue effect of interest arranged in a circle of six possible locations, corresponding to the 12:00, 2:00, 4:00, 6:00, 8:00, and 10:00 positions on a clock. In the no-cue and similarity-cue-only displays, one single-feature item appeared in each of the six possible locations. In the connection-only and two-cue displays, two possible stimulus arrangements were randomly intermixed: Either a single item appeared on top, with two items on the bottom, or two items appeared on top, with a single item on the bottom

(see the descriptions below), because this more clearly illustrated whether an effect was present and the direction of the effect; the results were the same as those from paired-sample $t$ tests (Fig. 3).

\section{Connection effect}

To determine whether connection groupings were used, performance in the no-cue condition was subtracted from that in the connection-only condition. A positive score reflected an improvement with connection cues. We found no connection effect for the color-only stimuli, $t(25)=0.53, p=.60, d=0.10$; however, significant positive connection effects emerged for 


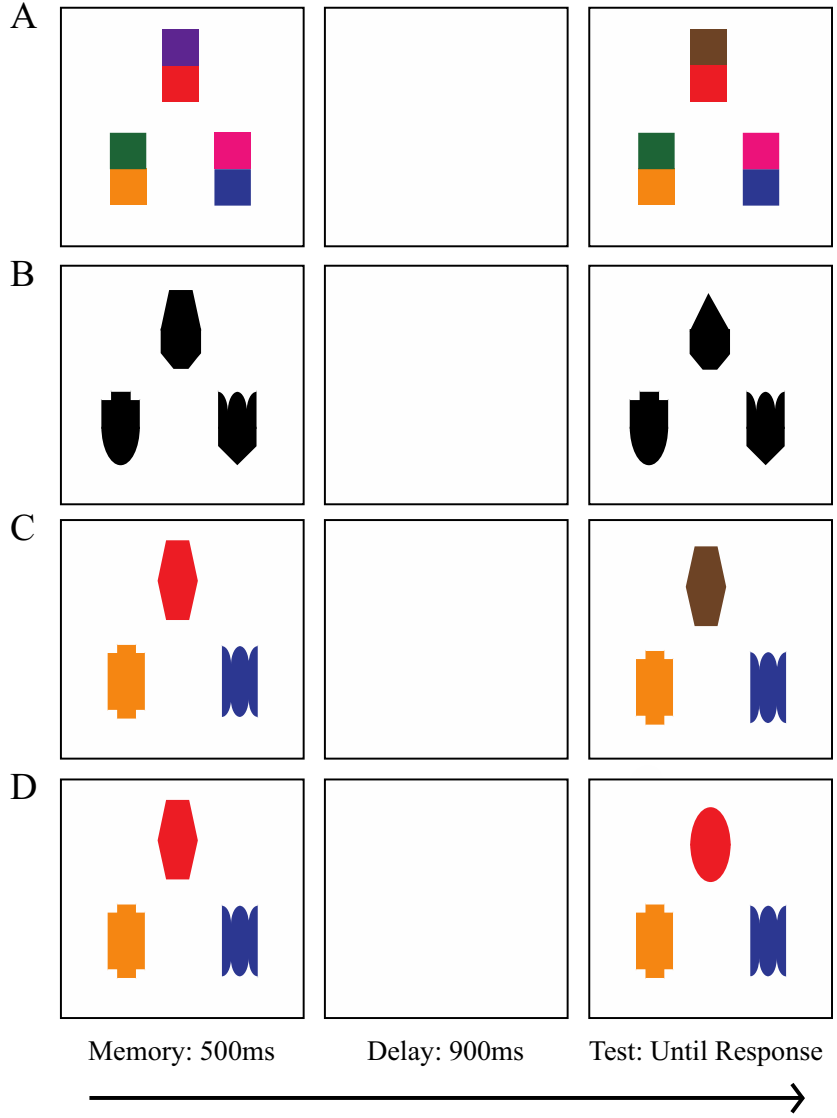

Fig. 2 Experimental procedure for Experiment 1. All four examples illustrate connection-only memory displays. (a) Color change for coloronly stimuli. (b) Shape change for shape-only stimuli. (c) Color change for color-and-shape stimuli. (d) Shape change for color-and-shape stimuli

the shape-only stimuli, $t(21)=3.58, p=.002, d=0.76$, and the color-and-shape stimuli, $t(20)=5.25, p<.001, d=1.15$.

\section{Similarity effect}

To determine whether similarity groupings were used, performance in the no-cue condition was subtracted from that in the similarity-only condition. A positive score reflected an improvement with similarity cues. Positive similarity effects were found for all stimulus types: color-only, $t(25)=3.76, p$ $=.001, d=0.74$; shape-only, $t(21)=2.03, p=.055, d=0.43$; color-and-shape, $t(20)=2.40, p=.03, d=0.52$.

\section{Connection effect for similar items}

To determine how connection cues affect similarity grouping, performance on the similarity-only trials was subtracted from that on the two-cue trials (two-cue - similarity-only). A positive value reflected improvement when connection cues were present, and a negative value reflected a detriment due to connection cues. When items were similar, there was a connection decrement for color-only stimuli, $t(25)=-2.63, p=$ $.02, d=-0.51$, and no connection effect for shape-only stimuli, $t(21)=2.03, p=.74, d=0.07$. However, we observed a significant positive connection effect for similar color-andshape stimuli, $t(20)=4.25, p<.001, d=0.93$.

\section{Similarity effect for connected items}

To determine how similarity affects connection grouping, performance on the connection-only trials was subtracted from that on the two-cue trials (two-cue - connection-only). This resulted in no similarity effect for connected color-only stimuli, $t(25)=-0.10, p=.93, d=-0.02$; a negative similarity effect for connected shape-only stimuli, $t(21)=-2.4, p=.03$, $d=-0.50$; and no similarity effect for connected color-andshape stimuli, $t(20)=1.3, p=.22, d=0.93$.

\section{Discussion}

The data show that connection and feature similarity cues can be used to increase VMW capacity. Specifically, connection can be used to improve memory for one color and one shape or two shapes, but not for two colors. The lack of a connection benefit for color-only stimuli is consistent with the previous literature (Delvenne \& Bruyer, 2004; Olson \& Jiang, 2002; Wheeler \& Treisman, 2002; Xu, 2002). However, the connection benefit for the shape-only and color-and-shape stimuli may rely on the connection creating a continuous surface feature, which was absent for the color-only stimuli. To test this, we conducted two control versions of the shape-only and colorand-shape conditions for which the connection did not create a continuous surface feature (see the supplemental material). Briefly, the data from these stimuli suggest that the shapeonly connection benefit may partially depend on the connection

Table 1 Summary of the results of Experiment 1

\begin{tabular}{|c|c|c|c|c|c|}
\hline \multirow[t]{2}{*}{ Stimulus Type } & \multirow[t]{2}{*}{ ANOVA } & \multicolumn{4}{|l|}{$M(S E)$} \\
\hline & & No-Cue & Connection-Only & Similarity-Only & Two-Cue \\
\hline Color-only & $F(3,75)=5.09, p=.003, \eta_{\mathrm{p}}^{2}=.17$ & $.67(.02)$ & $.68(.01)$ & $.74(.02)$ & $.68(.02)$ \\
\hline Shape-only & $F(3,63)=5.85, p=.001, \eta_{\mathrm{p}}^{2}=.22$ & $.60(.02)$ & $.67(.02)$ & $.64(.02)$ & $.63(.01)$ \\
\hline Color-and-shape & $F(3,60)=16.35, p<.001, \eta_{\mathrm{p}}{ }^{2}=.45$ & $.69(.01)$ & $.78(.02)$ & $.73(.02)$ & $.80(.03)$ \\
\hline
\end{tabular}




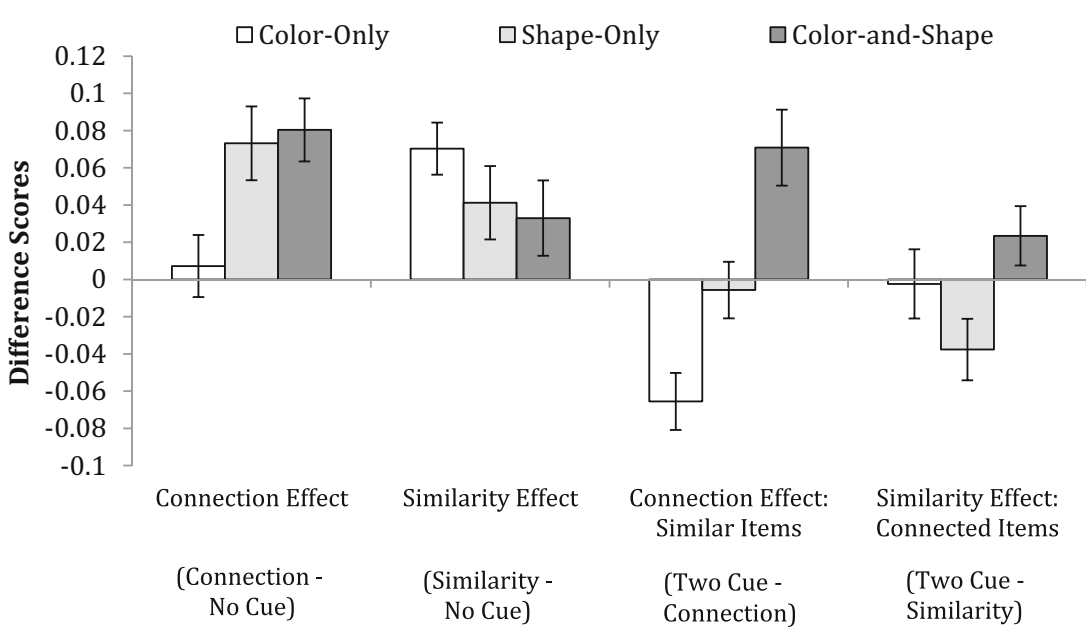

Fig. 3 Cue effects for each stimulus type. Error bars represent standard errors. A positive score reflects a benefit as a result of cue presence, whereas a negative score represents a detriment in performance due to cue presence

producing a continuous surface feature, but the color-and-shape connection benefit does not, which is consistent with the previous literature (Kim \& Kim, 2011; Xu, 2002).

Furthermore, the connection cue tends to dominate, particularly when the cues are in conflict. A connection benefit was present for similar color-and-shape stimuli, which suggests that color-shape connections may be more efficient at maximizing VWM capacity than similar features. Furthermore, although we found no connection benefit for color-color combinations, consistent with the previous literature (Delvenne \& Bruyer, 2004; Olson \& Jiang, 2002; Wheeler \& Treisman, 2002; Xu, 2002), performance was lower in the two-cue than in the similarity-only condition. This suggests that the connection cue eliminated the use of color similarity grouping, indicating that the connection cue is given preference when these two cues conflict.

Also, the presence of similar features reduced performance for the connected shapes, likely because the similarity created interference between the items (Johnson, Simmering, \& Buss, 2014; Wei, Wang, \& Wang, 2012). This suggests that the VWM representations may not be completely independent. Therefore, even though the connection cues may have precedence in VWM, similarity among the items can still affect how well each item is remembered. This is consistent with recent work demonstrating that scene statistics can influence the representations of single items (Brady \& Alvarez, 2011).

However, the most efficient grouping strategy for a given task may vary depending on the task goals. For example, if only color is task-relevant, then it would be more beneficial to group on the basis of color similarity across items than to group by color-and-shape connections. In Experiment 2, we investigated the extent to which the ability to select which grouping cue to use is under top-down control.

\section{Experiment 2}

Previous data had suggested it is possible to ignore taskirrelevant features and to selectively encode only the taskrelevant features into VWM (Kondo \& Saiki, 2012; van Lamsweerde \& Beck, 2011; Woodman \& Vogel, 2008). This suggests that the preferential use of a grouping strategy should be under top-down control. However, for the color-only stimuli of Experiment 1, participants only grouped by similarity when all colors were spatially separated, suggesting that connection cues may be particularly difficult to ignore.

To address the question of whether it is possible to control grouping strategies in VWM, the color-and-shape stimuli were used in Experiment 2. Participants detected color changes only, shape changes only, or randomly intermixed color and shape changes. When detecting a single change type (coloronly or shape-only), the optimal strategy would be to ignore the nonchanging dimension and to group by feature similarity in the task-relevant dimension. In contrast, when both dimensions can change, then the optimal strategy would be to group by color-and-shape connections in order to remember as many features as possible. If it is possible to flexibly switch between these connection and feature similarity grouping strategies, then there should be a similarity benefit when detecting a single change type, but not when detecting both changes.

\section{Method}

\section{Participants}

A total of 31 students (19 female, 12 male; average age = 20.23 years) at North Dakota State University participated in this experiment for course credit. Fourteen of the participants were assigned to the single-change group, and 17 participants were assigned to the both-change group. All participants 
reported normal or corrected-to-normal vision and normal color vision. One participant reported reversing the response keys; this was confirmed by the data, since this participant performed at $22 \%$ accuracy. Therefore, the data were reverse-scored for this participant and included in the analysis. The demographics reported here were calculated after the following participants were excluded: One reported starting off the first half of the experiment reversing the response keys, but then self-corrected partway through the experiment; the point at which the participant changed responses is unknown. Therefore, the data for this participant were rejected from the analysis. The data for two other participants were excluded because of computer failure before the experiment was over, which prevented them from completing the experiment.

\section{Stimuli and procedure}

In Experiment 2, we utilized the color-and-shape stimuli and the connection-only and two-cue displays from Experiment 1. In Experiment 2, lateralized displays were used and set size was manipulated (Fig. 4): Either three or four items were displayed on both the left and right sides of the screen. The memory display was preceded by an arrow cue indicating whether participants should attend to the left or the right hemifield for that trial (Vogel \& Machizawa, 2004). The colors and shapes were drawn for each side of the screen independently. Therefore, even when no feature repetition occurred within the cued side of the screen, there may have been repetition over the cued and uncued sides of the display (for either the changing or the nonchanging dimension). All participants completed both color and shape change trials; however, in the single-feature group these were separated into two counterbalanced blocks, and in the both-change group, the trials were randomly intermixed.

\section{Results}

In both change groups (single-feature and both-feature), the ANOVA results were the same for both change types (color and shape); therefore, the data presented here are for color and shape changes collapsed together. In each change group (single-feature, both-feature), a 2 (Set Size: three, four) $\times 2$ (Similarity Cue: similarity present, similarity absent) withinsubjects ANOVA was completed (Fig. 5). For both change groups, we observed a main effect of set size, as performance was better at set size 3 than 4 [single-feature: $F(1,13)=15.01$, $p<.002, \eta_{\mathrm{p}}{ }^{2}=.54$; both-feature: $F(1,16)=30.43, p<.001$, $\left.\eta_{\mathrm{p}}{ }^{2}=.66\right]$. In addition, in the single-feature group, a main effect of similarity cue was visible, since performance was better when the similarity cue was present than when it was absent $\left[F(1,13)=29.49, p<.001, \eta_{\mathrm{p}}{ }^{2}=.69\right]$. However, we found no effect of similarity cue in the both-feature condition $\left[F(1,16)=0.28, p=.61, \eta_{\mathrm{p}}{ }^{2}=.02\right]$. Furthermore, there was no Set Size $\times$ Similarity Cue interaction in any of the conditions [single-feature: $F(1,13)=0.61, p=.45, \eta_{\mathrm{p}}{ }^{2}=.05$; both-feature: $\left.F(1,16)=2.03, p=.17, \eta_{\mathrm{p}}{ }^{2}=.11\right]$. As in Experiment 1 , the data are presented as difference scores in order to more clearly demonstrate the similarity effect (similarity present similarity absent; the original means are presented in Table 2); a positive score represents an increase in performance due to similarity cue presence (see Fig. 5).

\section{Discussion}

Experiment 2 shows that it is possible to flexibly use either connection or feature similarity cues, depending on their task relevance. In Experiment 1, in which no similarity benefit occurred for connected color-and-shape items, participants detected both color and shape changes. In contrast, in Experiment 2, when only a single feature dimension was relevant (color-only and shape-only), participants improved with feature similarity cues. Replicating Experiment 1, when both feature dimensions were relevant (both-feature condition), no advantage was associated with feature similarity. This suggests that when only a single dimension was task-relevant, the irrelevant dimension was ignored and items were grouped by similarity along the task-relevant feature dimension.

Although the displays in Experiment 2 were somewhat different from those in Experiment 1, the overall performance indicated that participants were able to effectively ignore the

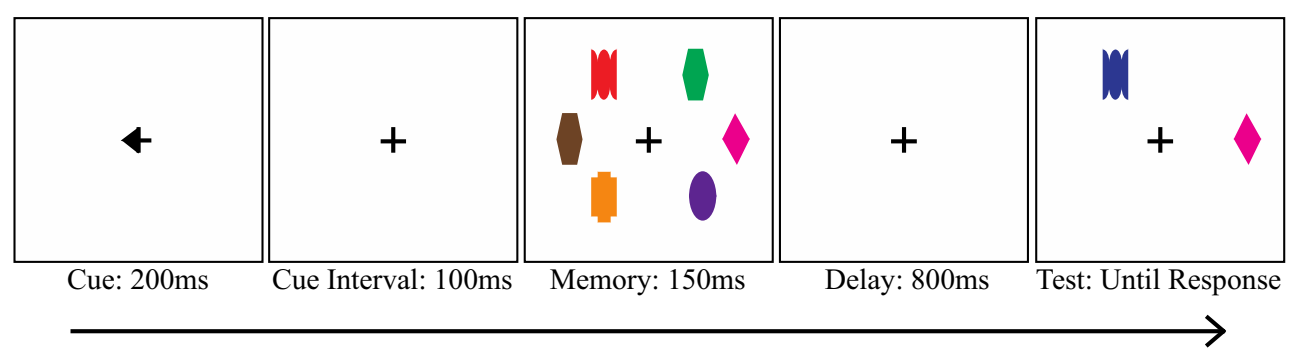

Fig. 4 Procedure for Experiment 2. Participants were cued to one visual hemifield and instructed to remember only those objects. A single object probe was used at test, and participants determined whether the probed object matched the item in that location from the memory display. The "test" item presented on the uncued side of the screen always matched the item from the memory display, and participants were instructed that this item was uninformative about the change on the cued side 


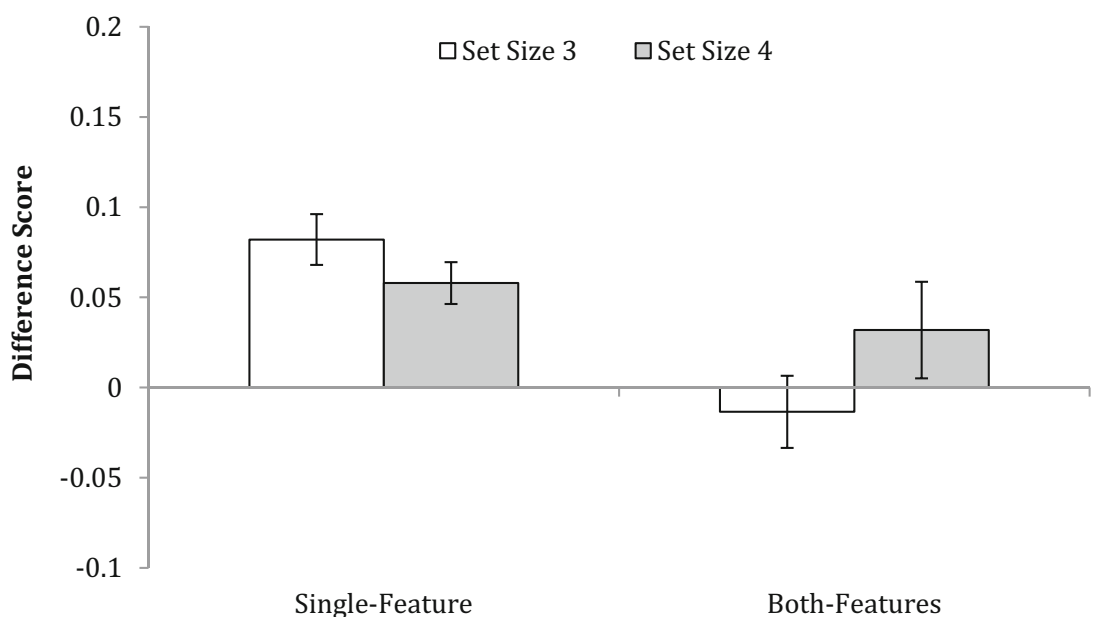

Fig. 5 Results of Experiment 2. Data are plotted as the difference between similarity-present and similarity-absent trials, with a positive score reflecting a benefit as a result of similarity cues. A similarity benefit

uncued side of the display. Specifically, overall performance in the both-feature, similarity-absent, set size 3 condition of Experiment $2(M=.75)$ was not different $(p=.36)$ from that in the equivalent condition (color-and-shape connection cue only) of Experiment $1(M=.78)$. This suggests that it is unlikely that the lateralized display design resulted in a failure to attend and encode any items in the cued hemifield as a result of attention spreading to the uncued hemifield. Furthermore, Experiment 2 replicated the similarity benefit with the new display design. Therefore, it appears that the ability to effectively utilize color-and-shape connections in VWM is consistent across different display types.

\section{General discussion}

These data show a connection between the organization of perception and subsequent VWM representations. Specifically, both connection and feature similarity can be used to group features and reduce VWM load. Furthermore, it is possible to flexibly choose which cue to use, depending on the goals of the task. However, whereas the perception of grouping two features together is likely dominated by

Table 2 Summary of the results of Experiment 2

\begin{tabular}{llll}
\hline Change Group & Set Size & \multicolumn{1}{l}{$M(S E)$} & \\
\cline { 3 - 4 } & & Similarity Present & Similarity Absent \\
\hline Single-feature & 3 & $.84(.03)$ & $.77(.03)$ \\
& 4 & $.77(.02)$ & $.72(.02)$ \\
Both-feature & 3 & $.73(.04)$ & $.75(.03)$ \\
& 4 & $.67(.03)$ & $.63(.02)$ \\
\hline
\end{tabular}

was found in the single-feature, but not in the both-feature, change condition. Error bars represent standard errors

connection, in some cases connection is not helpful in reducing VWM load. Although previous research has investigated how a variety of individual cues can be utilized by VWM (Luck \& Vogel, 1997; Peterson \& Berryhill, 2013; Woodman et al., 2003), this study expands our understanding of how information is organized in VWM by demonstrating the limits of certain cues to reduce VWM load, and that effective cue use depends on the presence of other, on conflicting cues, as well as on the task goals.

One goal of this study was to determine whether it is possible to flexibly shift between feature similarity and connection grouping cues. Experiment 1 demonstrated that it is possible either to use connection cues to group a color and a shape together, or to use feature similarity cues to group two identical colors or two identical shapes. Experiment 2 extended this and demonstrated that when both connection and feature similarity cues are present and in conflict, it is possible to flexibly switch between them, depending on which cue will better serve the task demands. Together, these data show that a range of grouping cues can be used to maximize VWM capacity, and it is possible to shift between them strategically.

However, there are situations in which the connection cue does not benefit VWM. First, in Experiment 1, we found no benefit for color-only connections. Furthermore, a benefit did emerge for the shape-only connections, but only when the connection created a continuous surface feature; disruption of this continuous surface feature eliminated the connection benefit (see the supplemental material). Therefore, connection may reduce shape VWM load only if it reduces the number of features that are perceived. That is, grouping itself is not sufficient to reduce VWM load in the case of shape-only stimuli; the grouping needs to produce fewer perceptible features. 
Therefore, although perceptually grouping features can reduce working memory load, connection alone is not always sufficient.

Although the connection does not always benefit VWM, it is a strong perceptual cue, and its presence can, in some cases, prevent the use of other grouping cues. Specifically, for the color-only stimuli, the presence of connection cues eliminated the feature similarity benefit, suggesting that it was not possible to ignore connection groupings in favor of feature similarity grouping. Of particular interest is why the connection cue was not ignored for the color-only stimuli, considering that it did not benefit VWM. If participants could flexibility shift between connection and feature similarity cues in Experiment 2, why could they not ignore color-color connections in favor of color similarity in Experiment 1? One possible explanation is that, unlike the color-and-shape stimuli in Experiment 2, in which a task-irrelevant feature dimension was suppressed (i.e., by ignoring color when only shape was relevant), all of the features of the coloronly stimuli in Experiment 1 were task-relevant. Therefore, attending to both features of the item may force participants to attend to the more dominant connection grouping. Alternatively, it is possible that participants were not given sufficient motivation for ignoring the connection grouping. With explicit instructions, participants might be able to ignore the connections and focus on grouping similar colors together.

We also found evidence that memory representations interact within VWM. In Experiment 1, performance for connected shape-only items was impaired when parts of the items were the same (e.g., the top halves were the same but the bottom halves were different), as compared to when all of the features were totally unique. This supports recent research demonstrating that VWM representations can influence one another, either by repulsion of similar items (Johnson et al., 2014) or by incorporating scene statistics into individual representations (Brady \& Alvarez, 2011). According to a strict "slot" view of VWM, each grouping in VWM consumes one unit's worth of storage (Luck \& Vogel, 1997). According to this view, it might be expected that representations would be maintained independently; however, the shape-only stimuli of Experiment 1 show that representations do affect each other in VWM.

The question of whether VWM is organized around objects (Awh, Barton, \& Vogel, 2007; Luck \& Vogel, 1997; Vogel, Woodman, \& Luck, 2001) or features (Bays, Wu, \& Husain, 2011; Fougnie \& Alvarez, 2011; Wheeler \& Treisman, 2002) suggests a static structure of VWM. However, the data here suggest that the organization of VWM is likely dynamic, depending on the available perceptual cues as well as top-down goals. An important issue for future research will be to determine the limits of the ability to flexibly shift between grouping strategies. For example, although it is possible to reduce the use of a perceptual grouping strategy through probabilities of its relevance (Beck \& Palmer, 2002), it does not appear that perception can be reorganized to the point of perceiving units that violate grouping cues (e.g., grouping two different colors together). Likewise, there may be limits to the ability to flexibly manipulate how features are grouped in VWM.

Acknowledgments This research was supported by the National Science Foundation (ND EPSCoR and NSF EPS-081444 to Jeffrey Johnson) and the National Institutes of Health (P20GM103505-08) to Mark McCourt.

\section{References}

Awh, E., Barton, B., \& Vogel, E. K. (2007). Visual working memory represents a fixed number of items regardless of complexity. Psychological Science, 18, 622-628. doi:10.1111/j.1467-9280. 2007.01949.x

Bays, P. M., Wu, E. Y., \& Husain, M. (2011). Storage and binding of object features in visual working memory. Neuropsychologia, 49, 1622-1631. doi:10.1016/j.neuropsychologia.2010.12.023

Beck, D. M., \& Palmer, S. E. (2002). Top-down influences on perceptual grouping. Journal of Experimental Psychology: Human Perception and Performance, 28, 1071-1084. doi:10.1037/0096-1523.28.5.1071

Brady, T. F., \& Alvarez, G. A. (2011). Hierarchical encoding in visual working memory: Ensemble statistics bias memory for individual items. Psychological Science, 10, 384-392. doi:10.1177/ 0956797610397956

Davis, G., \& Holmes, A. (2005). The capacity of visual short-term memory is not a fixed number of objects. Memory \& Cognition, 33, 185195. doi:10.3758/BF03195307

Delvenne, J.-F., \& Bruyer, R. (2004). Does visual short-term memory store bound features? Visual Cognition, 11, 1-27. doi:10.1080/ 13506280344000167

Erlikhman, G., Keane, B. P., Mettler, E., Horowitz, T. S., \& Kellman, P. J. (2013). Automatic feature-based grouping during multiple object tracking. Journal of Experimental Psychology: Human Perception and Performance, 39, 1625-1637. doi:10.1037/a0031750

Fougnie, D., \& Alvarez, G. A. (2011). Object features fail independently in visual working memory: Evidence for a probabilistic feature-store model. Journal of Vision, 11(12), 3:1-12. doi:10.1167/11.12.3

Goldfarb, L., \& Treisman, A. (2011). Does a color difference between parts impair the perception of a whole? A similarity between simultanagnosia patients and healthy observers. Psychonomic Bulletin \& Review, 18, 877-882. doi:10.3758/s13423-011-0123-8

Han, S. (2004). Interactions between proximity and similarity grouping: An event-related brain potential study in humans. Neuroscience Letters, 367, 40-43. doi:10.1016/j.neulet.2004.05.098

Han, S., Humphreys, G., \& Chen, L. (1996). Uniform connectedness and the classical Gestalt principles of grouping. Perception \& Psychophysics, 61, 661-674. doi:10.3758/BF03205537

Johnson, J. S., Simmering, V. R., \& Buss, A. T. (2014). Beyond slots and resources: Grounding cognitive concepts in neural dynamics. Attention, Perception, \& Psychophysics, 76, 1630-1654. doi:10. 3758/s13414-013-0596-9 
Kasai, T., Moriya, H., \& Hirano, S. (2011). Are objects the same as groups? ERP correlates of spatial attentional guidance by irrelevant feature similarity. Brain Research, 1399, 49-58.

Kim, S.-H., \& Kim, J.-O. (2011). The benefit of surface uniformity for encoding boundary features in visual working memory. Journal of Experimental Psychology: Human Perception and Performance, 37, 1767-1783.

Kondo, A., \& Saiki, J. (2012). Feature-specific encoding flexibility in visual working memory. PLoS ONE, 7(e50962), 1-8. doi:10.1371/ journal.pone.0050962

Luck, S. J., \& Vogel, E. K. (1997). The capacity of visual working memory for features and conjunctions. Nature, 390, 279-281. doi:10. $1038 / 36846$

Olson, I. R., \& Jiang, Y. (2002). Is visual short-term memory object based? Rejection of the "strong-object" hypothesis. Perception \& Psychophysics, 64, 1055-1067. doi:10.3758/BF03194756

Palmer, S., \& Rock, I. (1994). Rethinking perceptual organization: The role of uniform connectedness. Psychonomic Bulletin \& Review, 1, 29-55. doi:10.3758/BF03200760

Peterson, D. J., \& Berryhill, M. E. (2013). The Gestalt principle of similarity benefits visual working memory. Psychonomic Bulletin \& Review, 20, 1282-1289. doi:10.3758/s13423-013-0460-x

Schmidt, F., \& Schmidt, T. (2013). Grouping principles in direct competition. Vision Research, 88, 9-21. doi:10.1016/j.visres.2013.06.002

van Lamsweerde, A. E., \& Beck, M. R. (2011). The change probability effect: Incidental learning, adaptability, and shared visual working memory resources. Consciousness and Cognition, 20, 1676-1689. doi:10.1016/j.concog.2011.09.003

Vogel, E. K., \& Machizawa, M. G. (2004). Neural activity predicts individual differences in visual working memory capacity. Nature, 428 , 748-751. doi:10.1038/nature02447

Vogel, E. K., Woodman, G. F., \& Luck, S. J. (2001). Storage of features, conjunctions and objects in visual working memory. Journal of Experimental Psychology: Human Perception and Performance, 27, 92-114. doi:10.1037/0096-1523.27.1.92
Wannig, A., Stanisor, L., \& Roelfsema, P. R. (2011). Automatic spread of attentional response modulation along Gestalt criteria in primary visual cortex. Nature Neuroscience, 14, 1243-1244. doi:10.1038/nn.2910

Watson, S. E., \& Kramer, A. F. (1999). Object-based visual selective attention and perceptual organization. Attention, Perception, \& Psychophysics, 61, 31-49. doi:10.3758/ BF03211947

Wei, Z., Wang, X.-J., \& Wang, D. H. (2012). From distributed resources to limited slots in multiple-item working memory: A spiking network model with normalization. Journal of Neuroscience, 32, 11228-11240. doi:10.1523/JNEUROSCI. 0735-12.2012

Wertheimer, M. (1938). Laws of organization in perceptual forms. In W. Ellis (Ed.), A source book of gestalt psychology (pp. 71-88). London, UK: Kegan Paul. doi:10.1007/BF00410640

Wheeler, M. E., \& Treisman, A. M. (2002). Binding in short-term visual memory. Journal of Experimental Psychology: General, 13, 48-64. doi:10.1037/0096-3445.131.1.48

Woodman, G. F., Vecera, S. P., \& Luck, S. J. (2003). Perceptual organization influences visual working memory. Psychonomic Bulletin \& Review, 10, 80-87. doi:10.3758/BF03196470

Woodman, G. F., \& Vogel, E. K. (2008). Selective storage and maintenance of an object's features in visual working memory. Psychonomic Bulletin \& Review, 15, 223-229. doi:10.3758/PBR. 15.1.223

$\mathrm{Xu}$, Y. (2002). Limitations of object-based feature encoding in visual short-term memory. Journal of Experimental Psychology: Human Perception and Performance, 28, 458-468. doi:10.1037/00961523.28.2.458

$\mathrm{Xu}, \mathrm{Y}$. (2006). Understanding the object benefit in visual short-term memory: The roles of feature proximity and connectedness. Perception \& Psychophysics, 68, 815-828. doi:10.3758/ BF03193704

Zhang, W., \& Luck, S. J. (2009). Sudden death and gradual decay in visual working memory. Psychological Science, 20, 423-428. doi: 10.1111/j.1467-9280.2009.02322.x 